\title{
Eingeschüchtert und verunsichert - ReMed bietet Rückhalt
}

\section{Mirjam Tanner}

Dr. med., Mitglied des Leitungsausschusses von ReMed

Ärztinnen und Ärzte, denen fachlich ungerechtfertigte Fehler unterstellt und die vor Gericht gestellt werden, können unter dieser belastenden Lage leiden - selbst lange nach dem Freispruch. ReMed, das Unterstützungsnetzwerk für Ärztinnen und Ärzte in Krisensituationen, bietet Betroffenen professionelle Hilfestellung, um die Ängste abzubauen und die Sicherheit in die eigenen Fähigkeiten zurückzugewinnen.

\section{Allein auf sich gestellt}

«Der herablassende und blossstellende Ton in den Bemerkungen des anklagenden Anwalts während der Befragung - das war fast das Schlimmste an der ganzen Geschichte. Mit diesem Ton begann auch eine heftige und lang andauernde Verunsicherung und Verängstigung. Und dies, obwohl mir rational sehr klar war, dass der Anwalt Tatsachen willkürlich verdrehte und er mir fachlich definitiv keine Fehler vorwerfen konnte. Ich hatte gut verstanden: Diese berechnende, wuchtige Überzeugungskraft des Anklägers würde ihre Wirkung nicht nur auf mich haben.

Von nun an zitterte ich - still für mich - nicht bloss um den Verlust meiner Praxisbewilligung und die damit verbundenen sozialen und finanziellen Folgen. Schlimmer: Jeden Morgen beim Betreten meiner Praxis begleitete mich das Gefühl, dass ich eigentlich nichts kann, eine Versagerin bin und meine Patienten nicht adäquat behandle. Ausser mit dem Anwalt, der mich vor Gericht vertrat, konnte ich mit niemandem offen über meine Sorgen sprechen. Meine Ehe und Familie litten durch meine desolate psychische Verfassung massiv in diesen belastenden fünf Jahren. Ich schämte mich zu sehr, um professionelle Hilfe aufzusuchen, und behandelte mich als Hausärztin lieber selber mit Lexotanil und Antidepressiva.»

Erst rund zehn Jahre nach dem entlastenden Freispruch hat mir die Hausarztkollegin über ihre leidvolle Geschichte berichtet, in der ihr fachlich ungerechtfertigt ein Fehler unterstellt wurde und die ein jahrelanges Gerichtsverfahren zur Folge hatte. Sie fragt

\section{ReMed ist für Sie da}

Sind Sie oder jemand aus Ihrem Umfeld auf professionelle Hilfestellung angewiesen? Wenden Sie sich an ReMed: Das Unterstützungsnetzwerk für Ärztinnen und Ärzte respektiert das Arztgeheimnis und berät Sie kompetent. Auch bei anderen beruflichen und persönlichen Krisen kann ReMed Ihnen Lösungswege aufzeigen. Dieses Angebot ist auch für Personen aus dem Umfeld von Ärztinnen und Ärzten da, 24 Stunden am Tag; das Beraterteam meldet sich innerhalb von 72 Stunden: www.swiss-remed.ch, help[at]swiss-remed.ch, Tel. 0800 073633.

mich nun, was ReMed ihr damals in ihrem Fall hätte anbieten können.

\section{Austausch mit medizinischen Vertrauenspersonen}

ReMed ist eine niederschwellige Anlaufstelle, die Ärztinnen und Ärzte in Krisensituationen unterstützt. In einem ersten Kontakt klären sie gemeinsam mit einem Mitglied des Leitungsausschusses, welche Form der Unterstützung für sie die bestmögliche und passendste ist. Unsere Hausärztin hätte zum Beispiel durch eine supervisorische Begleitung in ihrem Praxisalltag schnell die Sicherheit für ihre fachliche Kompetenz zurückgewinnen können. Weiter kann es Betroffenen den Rücken stärken, wenn über langwierige, belastende und sehr verunsichernde Erfahrungen ein regelmässig begleitender Austausch mit einer medizinischen Vertrauensperson stattfinden kann. ReMed vermittelt 


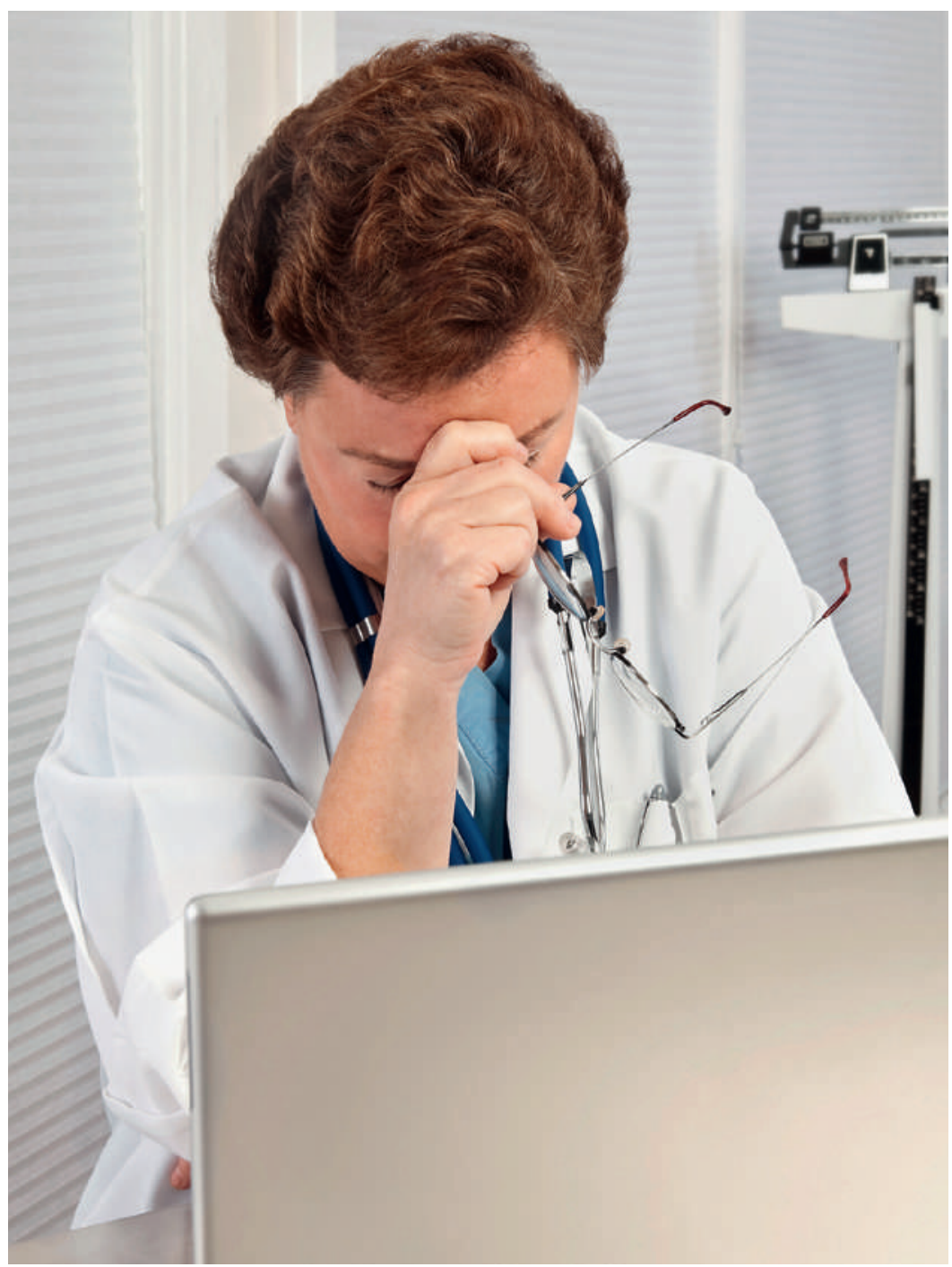

In einer schwierigen persönlichen Situation finden Ärztinnen und Ärzte bei ReMed verständnisvolle Unterstützung.

\section{Korrespondenz: \\ ReMed}

Sekretariat

Postfach 55

CH-3000 Bern 15

info[at]swiss-remed.ch hier gerne erfahrene und geeignete professionelle Unterstützung, beispielsweise aus dem Netz der Teilnehmenden der ReMed-Intervisionsgruppen.

Auch Ärztinnen und Ärzte können durch schwere Belastungen wie jahrelange juristische Auseinandersetzungen und die damit verbundene Ungewissheit

\section{Intervisionsgruppen: Termine 2015}

ReMed initiierte 2009 kollegiale Intervisionen, auch auf Wunsch von Kolleginnen und Kollegen. Seither organisiert das Unterstützungsnetzwerk regelmässig Peer-Groups (6-10 Teilnehmer, 2-3-mal/Jahr). Die Teilnehmenden erarbeiten gemeinsam ihre Fallfragen zu Mentoring, Coaching, Beratung, Therapie und anderen Aspekten kollegialer Begleitung (juristisch, versicherungsrechtlich usw.). Setzen Sie sich mit uns in Verbindung, nehmen Sie an einer Sitzung teil und lernen Sie unsere Arbeit kennen. Anmeldung über:

Dr. med. Peter Birchler, Tel. 0443420910 oder per Mail, peter. birchler[at]hin.ch

Restliche Daten für 2015: 25.6.2015 Hotel Central Plaza, Zürich 29.10.2015 Praxis Dolezal-Gasser, Aarau, 12.11.2015 Hotel Central Plaza, Zürich, 19.11.2015 Hotel Central Plaza, Zürich.

ein Trauma erleiden. Bei Bedarf bietet ReMed professionelle therapeutische Unterstützung an, sowohl in einer aktuell schwierigen Situation oder auch später. Aus vielen Gesprächen haben wir die Erkenntnis gewonnen, dass Kolleginnen und Kollegen, denen Fehler unterstellt und die in juristische Verfahren verwickelt wurden, selbst Jahre nach der Entlastung durch einen Freispruch geprägt waren vom oft sehr lang anhaltenden Druck, unter dem sie standen.

\section{Ängste als ständige Begleiter}

«Man spielt leicht den Druck herunter, den ein jahrelanges juristisches Verfahren durch die andauernde Verunsicherung auslöst. Irgendwann konnte ich die Ängste einfach nicht mehr aushalten, beispielsweise wenn sich beim Durchsehen der Post wieder ein Schreiben dieser Anwaltskanzlei befand und heftige Tachykardien und hypertone Ausnahmezustände einsetzten.

Aber es waren mehr als Angstsymptome und die Depression! Diese Geschichte bestimmte mein ganzes Leben und verfolgte mich den ganzen Tag auf Schritt und Tritt. Der ständige Versuch, sie wegzustecken, kostete mir unglaublich viel Kraft. Es wäre bereits hilfreich gewesen, wenn mich eine Kollegin professionell unterstützt und auch bezüglich Medikation hätte beraten können.» 